\title{
CORRECTIONS
}

\section{IDEAS ON KNOWLEDGE SYNTHESIS ..... a correction}

\author{
Donald Michie \\ The Turing Institute \\ Glasgow, Scotland
}

\author{
Ivan Bratko \\ E. Kardelj University and \\ Josef Stefan Institute \\ Ljubljana, Yugoslavia
}

We write to acknowledge a debt to the editors for their improvements to our paper "Ideas on Knowledge Synthesis Stemming from the KBBKN Endgame" (ICCA Journal, Vol. 10, No 1, pp. 3-13, March 1987). At the same time we wish to call attention to one point where an editorial change obscured our intended meaning.

The paper described the first two phases of an experiment on a leading chess endgame specialist's capacity to achieve mental mastery over the ultra-complex ending King and two Bishops against King and Knight (KBBKN). In phase 2 the specialist, Mr. A.J. Roycroft, was given interactive access to a complete tabulation of the raw facts of the domain, some two hundred million in all. Thus his task, as we may choose to express it, was to convert raw facts into effective operational concepts. His failure to do so to any significant extent invites explanation. We had in mind two alternatives.

Hypothesis (1). The complexity of the domain is such as to place the task beyond reach of all but those specialist professions whose members are trained to convert raw data into concepts (an "unnatural" aptitude, be it noted, scarcely ever required by the life or work of the rest of humanity, for whom inductive learning normally proceeds from data already possessing some degree of structure).

Hypothesis (2). The complexity of KBBKN is such that no human brain, however gifted or intensively trained for such a task, could perform the needed conversion.

Hypothesis (1) could then be tested by confronting one or more outstanding members of the cryptanalytic profession (code breakers), the example par excellence of raw-data-to-concepts conversion, with the same task under the same conditions as that faced by Roycroft.

Our original text said: "Whether a professional cryptanalyst or an empirical scientist placed under identical conditions would find himself equally at a loss is not known. These professions uniquely aim to incalculate a developed craft of converting raw facts to concepts". It would have been better for clarity to leave out "empirical scientist". Unfortunately the editors changed this to "trained methodologist", thus further obscuring the meaning.

We have no justified complaint, since we were given the opportunity to correct the amendment at proof stage, but missed it. The point however is so important, although essentially simple, that we will end by striking at it from another angle.

- If we wished to throw light on whether some given complex aero-engineering calculation is humanly feasible by unaided mental arithmetic, then after the world's leading aero-engineer had reported infeasibility we would surely try a domain specialist of another type, namely a professional calculating prodigy (the term "professional" is applicable since such specialists frequently earn their living by public performance on the stage). The calculating prodigy knows nothing about aeroplanes but is trained to perform seeming miracles of mental arithmetic.

- If we wished to throw light on whether a complex task of inductive theory extraction from a mountain of endgame facts is humanly feasible, then after the world's leading endgame specialist had reported infeasilibilty we should try a specialist of another type, namely a professional cryptanalyst. The cryptanalyst knows nothing about chess endgames but is trained to perform seeming miracles of induction from raw data. 